\title{
Intermittent recruitment with high-frequency oscillation/tracheal gas insufflation in acute respiratory distress syndrome
}

\author{
S.D. Mentzelopoulos*, S. Malachias*, E. Zintzaras \#, , S. Kokkoris*, E. Zakynthinos ${ }^{+}$, \\ D. Makris ${ }^{+}$, E. Magira*, V. Markaki*, C. Roussos* and S.G. Zakynthinos*
}

ABSTRACT: In acute respiratory distress syndrome (ARDS), recruitment sessions of highfrequency oscillation (HFO) and tracheal gas insufflation (TGI) with short-lasting recruitment manoeuvres (RMs) may improve oxygenation and enable reduction of subsequent conventional mechanical ventilation (CMV) pressures. We determined the effect of adding HFO-TGI sessions to lung-protective CMV on early/severe ARDS outcome.

We conducted a prospective clinical trial, subdivided into a first single-centre period and a second two-centre period. We enrolled 125 (first period, $n=54$ ) patients with arterial oxygen tension $\left(\mathrm{Pa}_{\mathrm{O}} \mathrm{O}_{2}\right)$ /inspiratory oxygen fraction $\left(\mathrm{FI}_{1} \mathrm{O}_{2}\right)$ of $<150 \mathrm{mmHg}$ for $>12$ consecutive hours at an end-expiratory pressure of $\geqslant 8 \mathrm{cmH}_{2} \mathrm{O}$. Patients were randomly assigned to an HFO-TGI group (receiving HFO-TGI sessions with RMs, interspersed with lung-protective CMV; $\mathbf{n}=61$ ) or CMV group (receiving lung-protective CMV and RMs; $n=64$ ). The primary outcome was survival to hospital discharge.

Pre-enrolment ventilation duration was variable. During days 1-10 post-randomisation, $\mathrm{Pa}, \mathrm{O}_{2} /$ $\mathrm{FI}_{1} \mathrm{O}_{2}$, oxygenation index, plateau pressure and respiratory compliance were improved in the HFOTGI group versus the CMV group ( $<<0.001$ for group $\times$ time). Within days $1-60$, the HFO-TGI group had more ventilator-free days versus the CMV group (median (interquartile range) 31.0 (0.0-42.0) versus $0.0(0.0-23.0)$ days; $\mathrm{p}<\mathbf{0 . 0 0 1 )}$, and more days without respiratory, circulatory, renal, coagulation and liver failure $(p \leqslant 0.003)$. Survival to hospital discharge was higher in the HFO-TGI group versus the CMV group (38 (62.3\%) out of 61 versus $23(35.9 \%)$ out of 64 subjects; $p=0.004)$. Intermittent recruitment with HFO-TGI and RMs may improve survival in early/severe ARDS.

KEYWORDS: Adult, clinical trial, high-frequency ventilation, respiratory distress syndrome

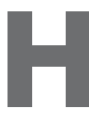
igh-frequency oscillation (HFO) is suggested for adults with severe acute respiratory distress syndrome (ARDS) [1, 2]. During HFO, tidal volumes of $<3.5 \mathrm{~mL} \cdot \mathrm{kg}^{-1}$ predicted body weight are administered at $\geqslant 3 \mathrm{~Hz}$ and mean airway pressure $\left(\bar{P}_{\text {aw }}\right)$ ranges $22-40 \mathrm{cmH}_{2} \mathrm{O}$ [1-3]. Animal lung injury data favour HFO over lung-protective conventional mechanical ventilation (CMV) [4]. The low HFO tidal volumes minimise volutrauma and the high HFO $\bar{P}_{\text {aw }}$ limits atelectrauma $[2,5]$.

When combined with 40-s recruitment manoeuvres (RMs), HFO improves oxygenation versus lung-protective $\mathrm{CMV}$, probably through lung recruitment [6-8]. The short-term addition of tracheal gas insufflation (TGI) to HFO may further improve oxygenation versus HFO without TGI and lung-protective CMV [7, 8]. TGI may promote lung recruitment by exerting a positive end-expiratory pressure (PEEP) effect and augmenting HFOdependent distal gas mixing [7-10].

We reasoned that a lung-protective, CMV-based ventilatory strategy employing extended (i.e. $\geqslant 6 \mathrm{~h}$ ) and repetitive (according to pre-specified criteria) recruitment sessions of HFO-TGI with RMs could result in a progressively sustained oxygenation improvement, with minimal concurrent risk of long-term HFO-TGI-related adverse effects [2, 7 , 10]. This should enable rapid reduction of subsequent CMV pressures to noninjurious levels [11]. A reduced lung end-inspiratory stretch could attenuate ventilator-associated lung injury $[12,13]$ and
AFFILIATIONS

*First Dept of Intensive Care Medicine, University of Athens Medical School, Athens,

"Depts of Biomathematics ${ }^{+}$Intensive Care Medicine, University of Thessaly Medical School, Larissa, Greece, and

- Center for Clinical Evidence Synthesis, The Institute for Clinical Research and Health Policy Studies, Tufts Medical Center, Tufts University School of Medicine, Boston, MA, USA.

\section{CORRESPONDENCE}

S.D. Mentzelopoulos

Evaggelismos General Hospital

45-47 Ipsilandou Street

GR-10675

Athens

Greece

E-mail: sdmentzelopoulos@

yahoo.com

Received:

Oct 102010

Accepted after revision:

Aug 152011

First published online:

Sept 012011 
improve outcome [14]. Thus, we compared the effect of two recruitment strategies during lung-protective $\mathrm{CMV}$, namely HFO-TGI sessions with short-lasting RMs versus short-lasting RMs alone, on the survival of patients with early/severe ARDS.

\section{METHODS}

\section{Patients}

The study was approved by the Scientific Committees of Evaggelismos Hospital (Athens, Greece) and Larissa University Hospital (Larissa, Greece). Informed, written next-of-kin consent was obtained for patients fulfilling the eligibility criteria presented in eTable 1 of the online supplementary material. Patients had early (onset within $\leqslant 72 \mathrm{~h}$ ) ARDS [15] and severe oxygenation disturbances: arterial oxygen tension $\left(\mathrm{Pa}_{3} \mathrm{O}_{2}\right)$ / inspiratory oxygen fraction $\left(\mathrm{FI}, \mathrm{O}_{2}\right)<150 \mathrm{mmHg}$ for $>12$ consecutive hours with a PEEP of $\geqslant 8 \mathrm{cmH}_{2} \mathrm{O}$; ARDS mortality increases at $\mathrm{Pa}_{1} \mathrm{O}_{2} / \mathrm{FI}_{1} \mathrm{O}_{2}<150 \mathrm{mmHg}$ [16]. We employed deep sedation and intermittent neuromuscular blockade with cisatracurium [12]. The sedation/paralysis and weaning (from CMV) protocols are detailed in the online supplementary material.

\section{Study design and randomisation}

We conducted a prospective, randomised, unblinded, parallelgroup controlled trial, temporally subdivided into a first singlecentre and a second two-centre period for feasibility reasons (online supplementary material). The 37-bed intensive care unit (ICU) of Evaggelismos Hospital participated during both periods. The 10-bed ICU of Larissa hospital participated in the second period. Following consent, patients were allocated to the intervention (HFO-TGI) or control (CMV) group according to computer-generated odd and even random numbers, respectively.
The HFO-TGI group received recruitment sessions of HFO-TGI with RMs according to pre-specified oxygenation criteria. HFOTGI sessions were interspersed with lung-protective CMV without RMs (table 1). The CMV group received lung-protective CMV and RMs for days 1-4 post-randomisation (table 1); the likelihood of sustained, RM-induced oxygenation improvement decreases and the risk of RM haemodynamic complications increases with CMV time [17]. In the HFO-TGI group, RMs were used after day 4 as part of the HFO-TGI protocol; RM-related oxygenation benefits are maintained when RMs are followed by HFO, even when HFO-time exceeds 4 days [6]. During days 1-4, minimum RM frequency was four per day in both groups. Figure 1 illustrates the study protocol.

\section{HFO-TGI recruitment protocol}

HFO was provided using a 3100B high-frequency ventilator (Sensormedics, Yorba Linda, CA, USA). The goal of each HFOTGI session was to increase $\mathrm{Pa}_{\mathrm{a}} \mathrm{O}_{2} / \mathrm{FI}_{1} \mathrm{O}_{2}$ to $>150 \mathrm{mmHg}$ by using a high initial $\bar{P}_{\text {aw }}$ (recruitment period), and then maintain the oxygenation benefit during a gradual $\bar{P}$ aw reduction to $6 \mathrm{cmH}_{2} \mathrm{O}$ below its initial value (stabilisation period) and during weaning from TGI and HFO (weaning period). Additional protocol features are described in online supplementary material.

Recruitment period: initial setting of HFO Paw

A rigid-wall catheter (inner diameter $1.0 \mathrm{~mm}$, outer diameter $2.0 \mathrm{~mm}$ ) was introduced during CMV. In each patient, catheter length was tailored to catheter tip placement at $0.5-1.0 \mathrm{~cm}$ beyond tracheal tube tip. CMV mean tracheal pressure $\left(\bar{P}_{\text {tr }}\right)$ was determined through the catheter with Direc218B (Raytech Instruments, Vancouver, Canada) over 3-min periods preceding transition to HFO. Patients were connected to the high-frequency ventilator and an RM was performed. Subsequently, a tracheal

TABLE 1 Conventional mechanical ventilation (CMV) strategy

Ventilator mode

Target tidal volume $\mathbf{m L} \cdot \mathbf{k g}^{-1}$ predicted body weight ${ }^{\#}$

Target end-inspiratory plateau pressure $\mathrm{cmH}_{2} \mathrm{O}$

Ventilator rate breaths $\cdot \min ^{-1} /$ target $\mathrm{pHa}$

Inspiratory-to-expiratory time ratio

Combinations of $\mathrm{Fl}, \mathrm{O}_{2} \% /$ PEEP $\mathrm{cmH}_{2} \mathrm{O}^{\circ}$

Target $\mathrm{Sp}_{\mathrm{p}} \mathrm{O}_{2} \%$

$\mathbf{R M}^{+, \mathbf{s}, f}$
Target $\mathrm{Pa}, \mathrm{O}_{2} \mathrm{mmHg}$

Volume assist control

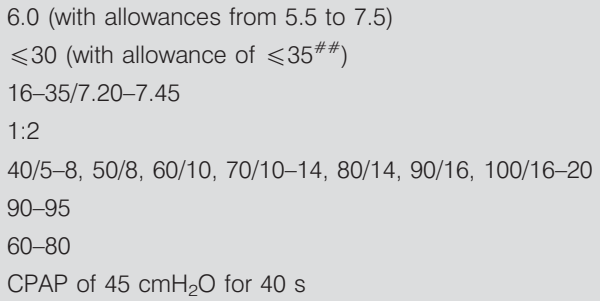

Apart from the protocolised use of recruitment manoeuvres (RMs), the presented CMV strategy mainly reflects standard clinical practice at both study centres. pHa: arterial blood $\mathrm{pH} ; \mathrm{Fl}_{1} \mathrm{O}_{2}$ : inspiratory oxygen fraction; $\mathrm{PEEP}$, positive end-expiratory pressure; $\mathrm{Sp}, \mathrm{O}_{2}$ : arterial oxygen saturation measured by pulse oximetry; $\mathrm{Pa}, \mathrm{O}_{2}$ : arterial

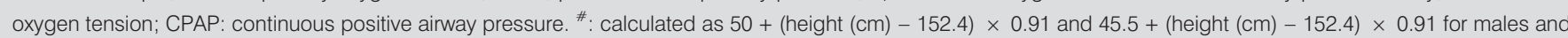
females, respectively. ": whenever the upper limit of the oxygenation targets was exceeded, PEEP was adjusted at a rate of $1-2 \mathrm{cmH}_{2} \mathrm{O} \cdot \mathrm{h}^{-1}$ (adjusting $\mathrm{Fl}, \mathrm{O}_{2}$ accordingly) an $\mathrm{Sp}, \mathrm{O}_{2}$ of $\leqslant 95 \%$ and/or an $\mathrm{Pa}_{1} \mathrm{O}_{2}$ of $\leqslant 80 \mathrm{mmHg}$ was reached; during the first 10 days post-randomisation, the downward titrations were reversed and suspended (for $12 \mathrm{~h}$ ) if 1) starting plateau pressure and $\mathrm{Fl}_{1} \mathrm{O}_{-2}$ were $\leqslant 30 \mathrm{cmH}_{2} \mathrm{O}$ and $\leqslant 70 \%$, respectively, and 2) they were associated with a $\mathrm{Pa}_{3} \mathrm{O}_{2} / \mathrm{Fl}_{1} \mathrm{O}_{2}$ decrease of $>25 \%$ and a $P \mathrm{a}_{1} \mathrm{O}_{2} /$ $\mathrm{Fl}_{1} \mathrm{O}_{2}$ of $<150 \mathrm{mmHg} .^{+}$: performed in the control, i.e. the CMV group, during the first 4 days after randomisation at a rate of one every 4-6 $\mathrm{h}$; post-RM PEEP was increased by $2 \mathrm{cmH}_{2} \mathrm{O}$, whenever the plateau pressure target of $30 \mathrm{cmH}_{2} \mathrm{O}$ was still achievable; within the following 60 min, if applicable, we re-titrated PEEP and $F \mathrm{l}, \mathrm{O}_{2}$ to the oxygenation targets as described. ${ }^{\varsigma}$ : in the intervention, i.e. the high-frequency oscillation (HFO) and tracheal gas insufflation (TGI) group, identical RMs were used solely during sessions of HFO-TGI (see Methods section and fig. 1). ${ }^{f}$ : in both groups, before each RM, we pre-oxygenated the patient by using an $\mathrm{Fl}, \mathrm{O}_{2}$ of $100 \%$ for $\geqslant 5$ min to reduce the risk of RM-associated desaturation [6]; for additional details, see the online supplementary material. ${ }^{\# \#}$ : whenever deemed necessary for achieving the lowest target $\mathrm{pHa}$ and/or $\mathrm{Sp}, \mathrm{O}_{2} / \mathrm{Pa}_{1} \mathrm{O}_{2}$; in such cases, tidal volumes of $5.5-6.0 \mathrm{~mL} \cdot \mathrm{kg}^{-1}$ were used. 
tube cuff-leak of $3-5 \mathrm{cmH}_{2} \mathrm{O}$ was placed and $\bar{P}_{\text {tr }}$ was remeasured. High-frequency ventilator-displayed $\bar{P}$ aw (HFO- $\left.\bar{P}_{\text {aw }}\right)$ was titrated to an $\mathrm{HFO}-\bar{P}_{\text {tr }}$ that exceeded preceding $\mathrm{CMV}-\bar{P}_{\mathrm{tr}}$ by $3 \mathrm{cmH}_{2} \mathrm{O}$. This resulted in an average HFO- $\bar{P}_{\text {aw }}$ of $8-9 \mathrm{cmH}_{2} \mathrm{O}$ above the preceding average $\mathrm{CMV}-\bar{P}_{\mathrm{aw}}$, because the average high-inspiratory flow-related drop [8] in HFO- $\bar{P}_{\text {aw }}$ along the tracheal tube was $\sim 6 \mathrm{cmH}_{2} \mathrm{O}$.

\section{TGl initiation}

Following setting of the initial HFO- $\bar{P}$ aw, the catheter was proximally connected to a variable-orifice oxygen flow meter providing pure, humidified oxygen at room temperature. Continuous, forward-thrust TGI was initiated through the catheter (TGI-flow $50 \%$ of preceding CMV minute ventilation [10]). TGI initiation caused a 1-2- $\mathrm{cmH}_{2} \mathrm{O}$ increase in HFO- $\bar{P}_{\text {aw }}$, which was reversed by adjusting the $\bar{P}_{\text {aw }}$ valve [10].

\section{Recruitment period duration}

If, at 60-90 min after HFO-TGI initiation, $\mathrm{Pa}_{1} \mathrm{O}_{2} / \mathrm{FI}_{1} \mathrm{O}_{2}$ exceeded $150 \mathrm{mmHg}$, we proceeded to the stabilisation period. Otherwise, the additional recruitment algorithm was applied, and the recruitment period extended until $\mathrm{Pa}, \mathrm{O}_{2} / \mathrm{FI}_{1} \mathrm{O}_{2}$ exceeded $150 \mathrm{mmHg}$ and/or $\bar{P}_{\text {aw }}$ reached $40 \mathrm{cmH}_{2} \mathrm{O}$ (fig. 1). The high-frequency ventilator $\mathrm{FI}_{1} \mathrm{O}_{2}$ was kept at $100 \%$ throughout this period.

\section{Stabilisation period: targeted HFO-P̄aw reduction}

$\bar{P}_{\text {aw }}$ was gradually reduced (rate $1-2 \mathrm{cmH}_{2} \mathrm{O} \cdot \mathrm{h}^{-1}$ ) to $3 \mathrm{cmH}_{2} \mathrm{O}$ below its initially set value. If $\mathrm{Pa}_{2} \mathrm{O}_{2} / \mathrm{FI}_{1} \mathrm{O}_{2}$ remained $>150 \mathrm{mmHg}$, an $\mathrm{RM}$ was performed and $\bar{P}_{\text {aw }}$ was decreased by another $3 \mathrm{cmH}_{2} \mathrm{O}$ at $1-2 \mathrm{cmH}_{2} \mathrm{O} \cdot \mathrm{h}^{-1}$. If $\mathrm{Pa}_{1} \mathrm{O}_{2} / \mathrm{FI}, \mathrm{O}_{2}$ was still $>150 \mathrm{mmHg}$, we proceeded to weaning period. Whenever these downward $\bar{P}_{\text {aw }}$ titrations resulted in a $\mathrm{Pa}, \mathrm{O}_{2} / \mathrm{FI}_{1} \mathrm{O}_{2}$ of $<150 \mathrm{mmHg}$, the additional recruitment algorithm was followed (fig. 1). The pre-specified minimum duration of the stabilisation period was $240 \mathrm{~min}$.

Ventilator $\mathrm{FI}, \mathrm{O}_{2}$ was reduced to 80,70 or $60 \%$ if the $\mathrm{Pa}_{2} \mathrm{O}_{2} / \mathrm{FI}, \mathrm{O}_{2}$ of the immediately preceding physiological measurement was 150-200, 200-300 or $>300 \mathrm{mmHg}$, respectively. Prior to and during each subsequent physiological measurement, ventilator $\mathrm{FI}_{1} \mathrm{O}_{2}$ was set at $100 \%$ (for $20 \mathrm{~min}$ ). This enabled precise determination of $\mathrm{Pa}_{\mathrm{a}} \mathrm{O}_{2} / \mathrm{FI}, \mathrm{O}_{2}$ during ongoing TGI.

\section{Weaning period: discontinuation of TGI and HFO}

An RM was performed and TGI was discontinued over $30 \mathrm{~min}$; the associated HFO- $\bar{P}$ aw reduction of $1-2 \mathrm{cmH}_{2} \mathrm{O}$ was reversed by adjusting the $\bar{P}_{\text {aw }}$ valve. Patients were ventilated with standard $\mathrm{HFO}$ for a further $30 \mathrm{~min}$ and if $\mathrm{Pa}, \mathrm{O}_{2} / \mathrm{FI}_{1} \mathrm{O}_{2}$ was $>150 \mathrm{mmHg}$, they were returned to CMV. If $\mathrm{Pa}, \mathrm{O}_{2} / \mathrm{FI}_{1} \mathrm{O}_{2}$ was $<150 \mathrm{mmHg}$, patients were returned to the additional recruitment algorithm (fig. 1).

\section{HFO-TGI session duration}

The minimum time from HFO initiation to HFO termination was $6 \mathrm{~h}$. Each transition to the additional recruitment algorithm (fig. 1) extended the session by $\geqslant 2-3 \mathrm{~h}$. After every $12-$ $24 \mathrm{~h}$ of HFO-TGI, a brief bronchoscopic inspection of the carina was performed to rule out TGI-induced tracheal mucosal damage.

\section{Return to HFO-TGI}

The criterion for return to HFO-TGI was $\mathrm{Pa}_{\mathrm{a}} \mathrm{O}_{2} / \mathrm{FI}_{1} \mathrm{O}_{2}<150 \mathrm{mmHg}$ sustained for $>12$ consecutive hours, while on CMV. Patients were assessed for return to HFO-TGI at 12 and $24 \mathrm{~h}$ after return to $\mathrm{CMV}$, and then at the beginning of each day until day 10 postrandomisation.

\section{Definitions}

Definitions of organ/system failures according to a corresponding Sequential Organ Failure Assessment (SOFA) subscore $\geqslant 3$ [18], infections and other complications are provided in the online supplementary material. Multiple organ failure (MOF) was defined as three or more concurrent organ/system failures [19].

\section{Follow-up}

Baseline patient data were recorded within $2 \mathrm{~h}$ pre-randomisation. Daily recordings included physiological/laboratory data (days 1-28 post-randomisation), intervention-associated complications (days 1-10; e.g. RM-induced hypotension or desaturation), mechanical ventilation-associated barotrauma (study-independent radiologists assessed chest radiographs for pathological gas collection(s), e.g. pneumothorax), data on organ/system failures and medication (days 1-60), episodes of failure to maintain unassisted breathing and various complications (until hospitaldischarge or death; e.g. infections and heparin-induced thrombocytopenia). Investigators were unblinded to patient outcomes. Adherence to the protocol was overseen by the Data Monitoring Committee (see Acknowledgements section for details).

During days 1-10, sets of physiological measurements were obtained as follows. 1) CMV group: three measurements per day, starting at 09:00 h. 2) HFO-TGI group: just before, during and $6 \mathrm{~h}$ after HFO-TGI, and as in CMV group if no longer requiring HFO-TGI. Measurements included arterial/centralvenous blood-gas analysis, haemodynamics and respiratory mechanics while on CMV [7, 12]. For between-group comparisons, we used CMV data obtained between 09:00 and 10:00 h in both groups.

\section{Outcome measures}

\section{Primary}

The primary outcome was survival to hospital-discharge, i.e. "patient discharged home, while breathing without assistance."

\section{Secondary}

The secondary outcomes were: ventilator-free and organ/ system failure-free days up to day 28 and 60, i.e. follow-up days within days 1-28 and 1-60, minus days on a ventilator or days with organ/system failure (for survivors, minimum follow-up was 60 days); mechanical ventilation-associated barotrauma; TGI-related tracheal mucosal injury; and evolution of oxygenation, plateau pressure and respiratory compliance during the period of HFO-TGI use.

\section{Statistical analysis}

Additional details are provided in the online supplementary material. According to the pilot cohort data, the predicted survival rate to hospital discharge was 66 and $40 \%$ for the HFO-TGI group and CMV group, respectively. For an $\alpha$-value of 0.05 and a power of 0.80 , a total sample size of 124 patients was required. Interim analyses were conducted at the completion of the follow-up of the 84th and 104th patient; stopping rules were $\mathrm{p}<0.001$ for efficacy and $\mathrm{p}>0.1$ for futility. All study personnel were masked from interim analyses results. 


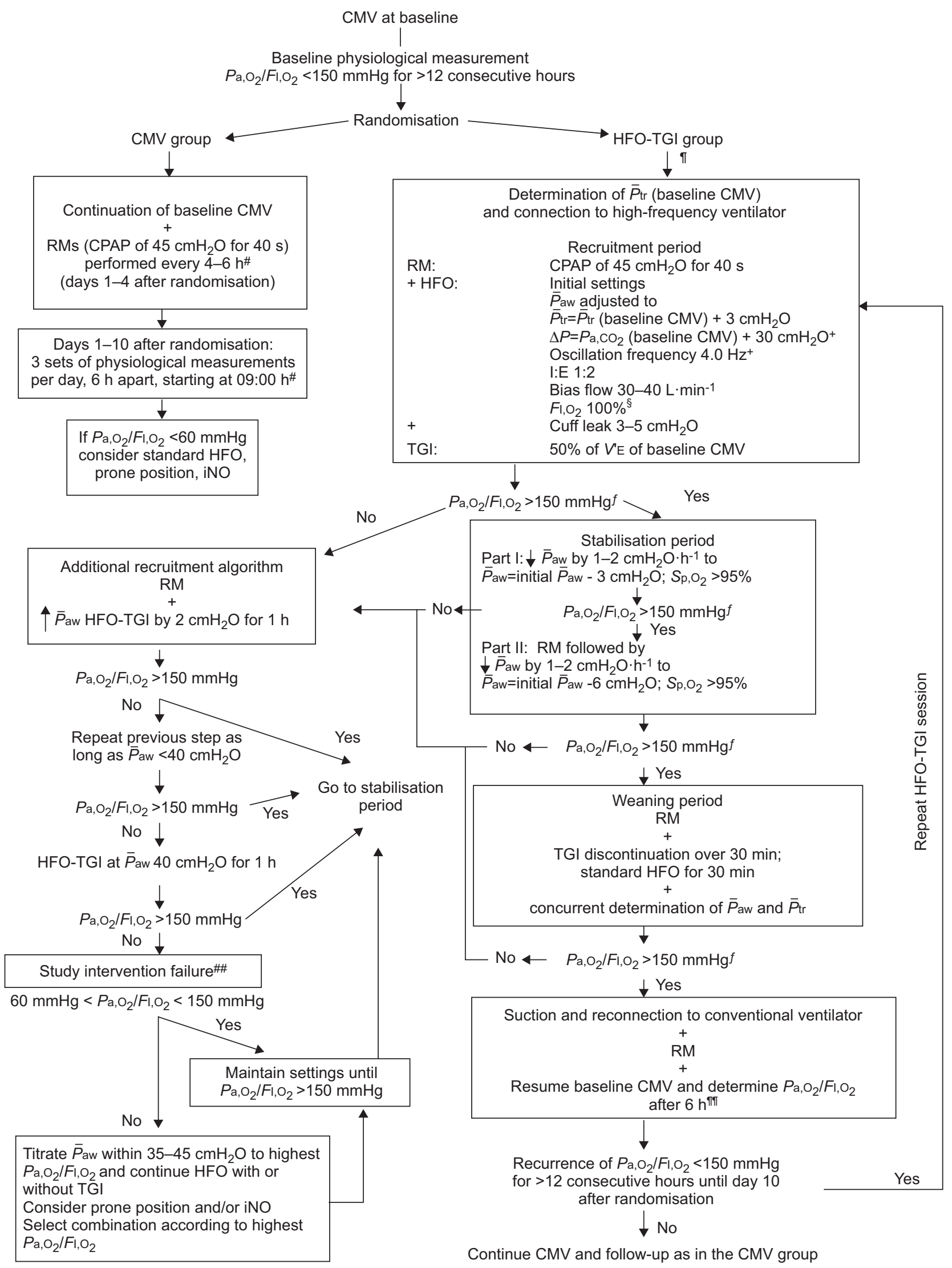


FIGURE 1. Algorithmic representation of the study protocol. During high-frequency oscillation (HFO) tracheal gas insufflation (TGI), recruitment manoeuvres (RMs) were performed with TGl turned off and the tracheal tube cuff inflated. Target arterial oxygen saturation measured by pulse oximetry $\left(\mathrm{Sp}, \mathrm{O}_{2}\right)$ was $>95 \%$ for all periods (see online supplementary material); at any period, an $\mathrm{Sp}, \mathrm{O}_{2}$ of $<88 \%$ for $>5 \mathrm{~min}$ was the trigger for immediate transition to the additional recruitment algorithm, or to its next step if the desaturation occurred during its application. Note that any transition to the additional recruitment algorithm resulted in mean airway pressure $\left(\bar{P}_{\text {aww }}\right)$ increase of $\geqslant 2 \mathrm{~cm} \mathrm{H}_{2} \mathrm{O}$, which had to be reversed after the subsequent transition to the stabilisation period. This resulted in extension of the stabilisation period by $\geqslant 1 \mathrm{~h}$. CMV: conventional mechanical ventilation; $\mathrm{Pa}_{\mathrm{a}, \mathrm{O}_{2}}$ : arterial oxygen tension; $\mathrm{Fl}, \mathrm{O}_{2}$ : inspiratory oxygen fraction; CPAP: continuous positive airway pressure; iNO: inhaled nitric oxide; $\bar{P}_{\mathrm{tr}}$ : mean tracheal pressure; $\triangle P$ : oscillatory pressure amplitude; $\mathrm{Pa}_{1} \mathrm{CO}_{2}$ : arterial carbon dioxide tension; I:E, inspiratory-to-expiratory time ratio; $V^{\prime} \mathrm{E}$ : minute ventilation. ${ }^{\#}$ : temporal distance between any measurement and a preceding RM was $\geqslant 2 \mathrm{~h} .{ }^{\top}$ : corresponds to the timing of the first set of daily physiological measurements performed during CMV, prior to HFO-TGI initiation; by design, these measurements were to be performed at 09:00 $\mathrm{h}$ unless the patient was already on HFO-TGI at that particular time (see online supplementary material). ${ }^{+}$: frequency and $\Delta P$ were adjusted to maintain an arterial blood $\mathrm{pH}$ of $\geqslant 7.20$ by means of two consecutive arterial blood gas analyses performed within the first 30 min of the recruitment period. ${ }^{\varsigma}$ : high-frequency ventilator $\mathrm{Fl}, \mathrm{O}_{2}$ was initially set at $100 \%$; for further details regarding the management of $\mathrm{Fl}, \mathrm{O}_{2}$ see the Methods section. ${ }^{f}$ : corresponds to the timing of physiological measurements during the HFO-TGl session; in HFO-TGI sessions exceeding 6 h, one additional set of physiological measurements was obtained for every additional 2-4 h. \#\#: the corresponding, pre-specified management technique is presented in detail in the subsection "Additional features of the HFO-TGI protocol" of the eMethods in the online supplementary material; RMs were not performed at $\bar{P}_{\text {aw }}>40 \mathrm{cmH}_{2} \mathrm{O}$; one RM was performed every $1-2 \mathrm{~h}$ during periods with $\bar{P}_{\text {aw }}$ of $35-40 \mathrm{cmH}_{2} \mathrm{O} .{ }^{\prime}{ }^{\prime}$ : corresponds to the timing of physiological measurements performed during CMV, after weaning from TGl and $\mathrm{HFO}$.

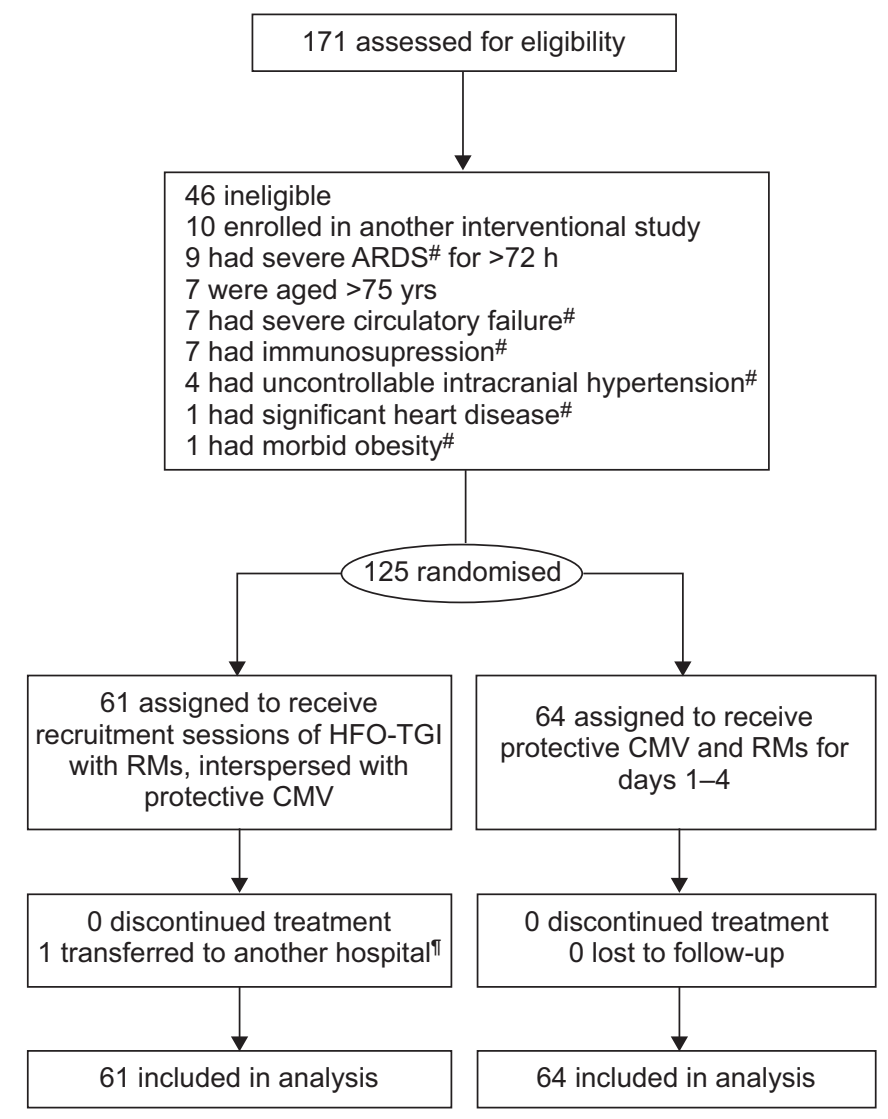

FIGURE 2. Study flow chart. ARDS: acute respiratory distress syndrome; HFO: high-frequency oscillation; TGI: tracheal gas insufflation; RM: recruitment manoeuvre; CMV: conventional mechanical ventilation. ${ }^{*}$ : definition provided in the footnote of eTable 1 in the online supplementary material; additional pre-specified exclusion criteria not met by anyone of the 171 potentially eligible patients were active air leak or recent severe air leak, obstructive or interstitial lung disease, lung surgery on current admission, pregnancy, and dependency on prone positioning or inhaled nitric oxide (eTable 1); the lower limits for age and body weight were $18 \mathrm{yrs}$ and $40 \mathrm{~kg}$, respectively. ": the patient was transferred to another hospital not participating in the study on day 31 post-randomisation; the data from this patient were included in the intention-to-treat analysis, assuming that he died, because at the time-point of transfer the patient was not discharged home and was not breathing without assistance (see the main text for the definition of "hospital discharge").
An intention-to-treat analysis was performed with SPSS version 12.0 (SPSS, Chicago, IL, USA) and SAS version 9.0 (SAS Institute, Cary, NC, USA). Data are reported as mean \pm SD, median (interquartile range) or $\mathrm{n}(\%)$, unless otherwise specified. Dichotomous and categorical variables were compared using Fisher's exact test. Continuous variables were compared using a twotailed, independent-samples t-test or the Mann-Whitney exact U-test. The Bonferroni correction was used for multiple comparisons. For days $1-10$, the effects of group, time and group $\times$ time on physiological variables were determined by mixed-model analysis. Survival was analysed with the Kaplan-Meier method, and survival data were compared by Fisher's exact test and the log-rank test. Cox regression was used to determine independent predictors of death. The effect of centre was assessed by betweencentre comparisons for study end-points. Reported p-values are two-sided. Significance was accepted at $\mathrm{p}<0.05$.

\section{RESULTS}

The study was conducted from July 1, 2006 to September 29, 2007 (first period; $\mathrm{n}=54$ ) and from March 10, 2008 to May 30, 2009 (second period; $\mathrm{n}=71$ ). From 171 potentially eligible patients, 125 were randomised (HFO-TGI group, $n=61$; CMV group, $n=64$ ) and their data analysed (fig. 2). 16 (34.8\%) out of the 46 excluded patients survived to hospital discharge.

Table 2 presents baseline characteristics. 85 (68.0\%) patients (HFO-TGI group, $n=40$ ) had MOF. The HFO-TGI intervention period extended to day 10 post-randomisation. Table 3 presents data on daily HFO-TGI; session duration ranged 6.0-102.2 h.

\section{Physiological variables during intervention period}

Physiological variables during the intervention period are summarised in table 4 . There were no significant between-group differences in haemodynamics, arterial blood lactate or haemodynamic support. Measures of oxygenation $\left(\left(\mathrm{Pa}, \mathrm{O}_{2} / \mathrm{FI}_{1} \mathrm{O}_{2}\right)\right.$ and oxygenation index) and lung mechanics (plateau pressure and respiratory compliance) improved substantially over days 1-10 in the HFO-TGI group (table 4 and fig. 3a-d).

\section{Response to HFO-TGI}

Mean \pm SD pre-session $\mathrm{Pa}_{2} \mathrm{O}_{2} / \mathrm{FI}_{1} \mathrm{O}_{2}$ rose from $110.6 \pm 32.0$ to $256.1 \pm 93.1 \mathrm{mmHg}$ during the recruitment period (maximum duration $8.5 \mathrm{~h}$ ). Oxygenation improvement was primarily due to the high $\bar{P}_{\text {aw }}$, RMs and TGI (fig. 1) $[7,8]$. Subsequently, $\mathrm{Pa}_{2} \mathrm{O}_{2} / F \mathrm{I}, \mathrm{O}_{2}$ 
TABLE 2 Patient characteristics just prior to randomisation

\begin{tabular}{|c|c|c|}
\hline & HFO-TGI group & CMV group \\
\hline Subjects n & 61 & 64 \\
\hline Males & $46(75.4)$ & $47(73.4)$ \\
\hline Body mass index $\mathrm{kg} \cdot \mathrm{m}^{-2}$ & $26.4 \pm 4.3$ & $25.4 \pm 2.5$ \\
\hline Predicted body weight ${ }^{\#} \mathbf{k g}$ & $71.2 \pm 8.9$ & $69.7 \pm 7.7$ \\
\hline Diabetes mellitus & $7(11.5)$ & $7(10.9)$ \\
\hline Neoplasm & $7(11.5)$ & $9(14.1)$ \\
\hline Other $^{+}$ & $5(8.2)$ & $6(9.4)$ \\
\hline \multicolumn{3}{|l|}{ Surgical operations during current hospitalisation } \\
\hline Major elective operation & $5(8.2)$ & $10(15.6)$ \\
\hline Tracheotomised before enrolment & $8(13.1)$ & $8(12.5)$ \\
\hline SAPS II (predicted death rate \%) & $43.5 \pm 12.3(35.2 \pm 23.4)$ & $43.6 \pm 10.9(34.4 \pm 20.2)$ \\
\hline Presence of $\geqslant 2 / \geqslant 3$ organ/system failures & $61(100.0) / 40(65.6)$ & $63(98.4) / 45(70.3)$ \\
\hline \multicolumn{3}{|l|}{ Circulatory failure } \\
\hline Septic aetiology & $46(75.4)$ & $46(71.9)$ \\
\hline Nonseptic aetiology & $9(14.8)$ & $11(17.2)$ \\
\hline Primary ARDS & $50(82.0)$ & $50(78.1)$ \\
\hline \multicolumn{3}{|l|}{ Aetiology of ARDS } \\
\hline Hospital-acquired pneumonia ${ }^{f, \# \#, \text {, }}$ & $20(32.8)$ & $22(34.4)$ \\
\hline Community-acquired pneumonia ${ }^{++}$ & $7(11.5)$ & $10(15.6)$ \\
\hline Bilateral pulmonary contusions $\# \#, \S \S$ & $22(36.1)$ & $12(18.9)$ \\
\hline Polytransfusion $\bullet, \S \S$ & 5 (8.2) & $8(12.5)$ \\
\hline
\end{tabular}

Data are presented as mean \pm SD, $n$ (\%) or median (interquartile range), unless otherwise stated. HFO: high-frequency oscillation; TGl: tracheal gas insufflation; CMV: conventional mechanical ventilation; DMV: duration of mechanical ventilation; ALI: acute lung injury; ARDS: acute respiratory distress syndrome; ICU: intensive care unit; SAPS: Simplified Acute Physiology Score. ${ }^{*}$ : the corresponding calculation formula is provided in the footnote of table 1. ": some patients had more than one comorbid condition. ${ }^{+}$: includes six cases of alcohol abuse (three in each group), two cases of chronic atrial fibrillation (one in each group), two cases of intravenous drug abuse (one in each group), two cases of major depression (one in each group), and one case each of cerebrovascular disease (HFO-TGl group) and schizophrenic disorder (CMV group). "s: on ICU admission, all patients were receiving mechanical ventilation for acute respiratory failure; also, within 1, 24 and $36 \mathrm{~h}$ of ICU admission, 103 , seven and three patients, respectively, were confirmed as fulfilling the criteria for ALI/ARDS [15]; pre-enrolment DMV reflects time elapsed from DMV initiation to the onset of ALI/ ARDS plus time elapsed from the latter time-point to the time-point of the fulfilment of the present study's criterion for severe oxygenation disturbance plus the time needed to obtain next-of-kin consent (see also text); pre-enrolment DMV was $<7$ days in 53 (86.9\%) patients of the HFO-TGl group and 60 (93.8\%) patients of the CMV group. ${ }^{f}$ : caused by Acinetobacter baumannii (12 cases in the HFO-TGI group and 11 cases in the CMV group), Klebsiella pneumoniae (six cases in the HFO-TGI group and six cases in the CMV group) and Pseudomonas aeruginosa (two cases in the HFO-TGI group and five cases in the CMV group); 17 HFO-TGl group patients and 20 CMV group patients had concurrent circulatory failure. ${ }^{\# \#}$ : both factors were simultaneously present in seven patients in the HFO-TGI group and two patients in the CMV group. ": Both factors were simultaneously present in one patient in the HFO-TGI group. ${ }^{++}$: caused by Streptococcus pneumoniae (two cases in the HFO-TGI group and four cases in the CMV group), Legionella pneumophila (three cases in the HFO-TGl group and two cases in the CMV group), methicillin-resistant Staphylococcus aureus (one case in the HFO-TGI group and two cases in the CMV group) and $K$. pneumoniae (one case in the HFO-TGl group); one case in the CMV group was of unknown aetiology; six HFO-TGl group patients and eight CMV group patients had concurrent circulatory failure. ${ }^{\S \S}$ : both factors were simultaneously present in one patient of the HFO-TGI group and one patient of the CMV group. ${ }^{f f}$ : includes three cases of acute interstitial pneumonia (one in the HFO-TGI group and two in the CMV group), three cases of necrotising fasciitis (one in the HFO-TGI group and two in the CMV group; one patient from each group also received a massive blood transfusion), two cases of thermal injury (one in each group), and one case each of urosepsis due to Proteus mirabilis (HFO-TGl group), sepsis due to Serratia marcescens (HFO-TGI group), cerebral ventriculitis and sepsis due to A. baumannii (HFO-TGI group), necrotising Gram-negative pneumonia (HFO-TGl group; the patient also had bilateral pulmonary contusions), necrotising pancreatitis (CMV group; the patient had also suffered an episode of pulmonary aspiration), alveolar haemorrhage (CMV group), submersion injury (HFO-TGI-group), toxic epidermal necrolysis (HFO-TGI group; the patient also had aspiration pneumonia), post-operative mediastinitis (CMV group) and surgical wound infection (CMV group). 


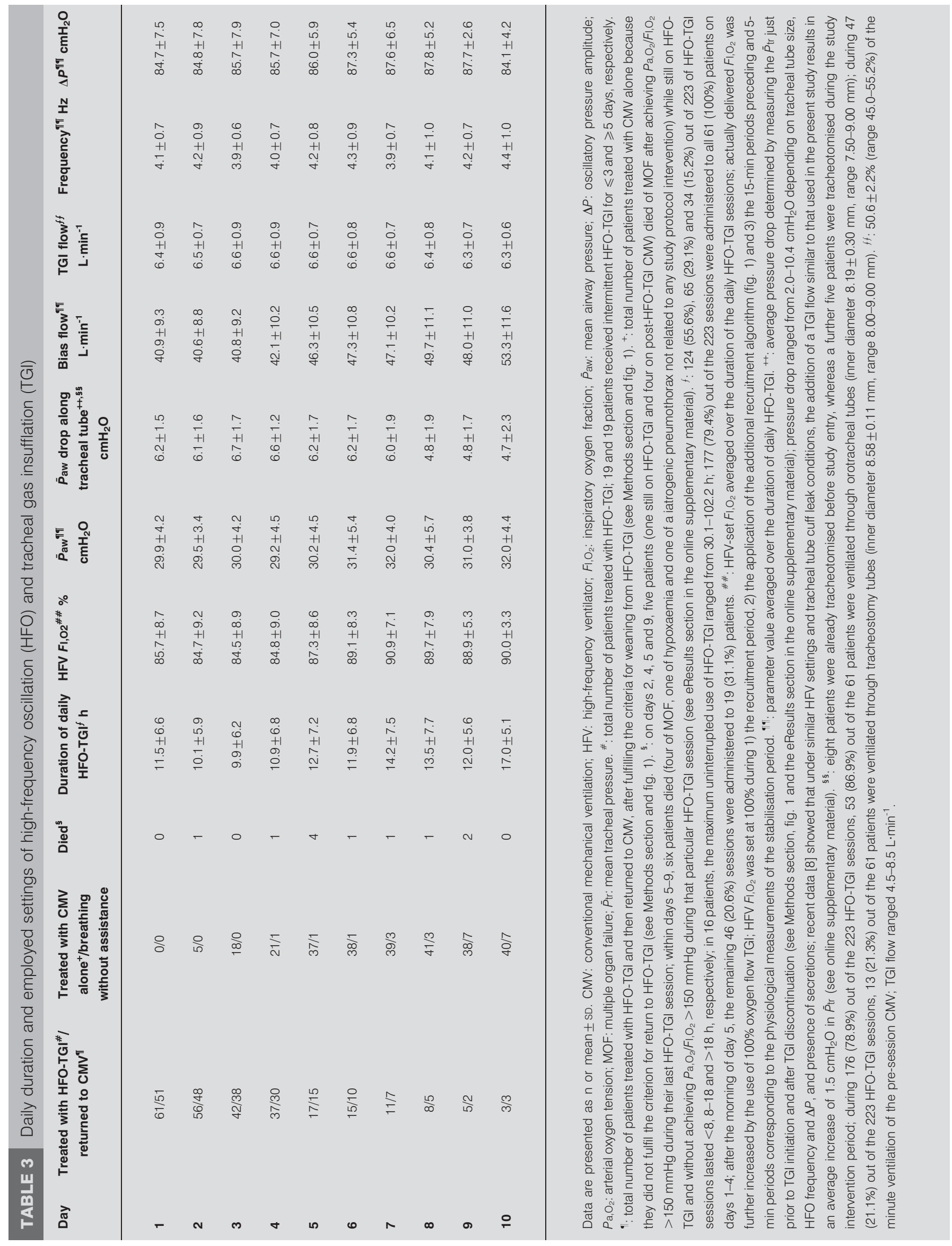


TABLE 4 Physiological variables, haemodynamic support and organ failure assessment for days 1-10 post-randomisation

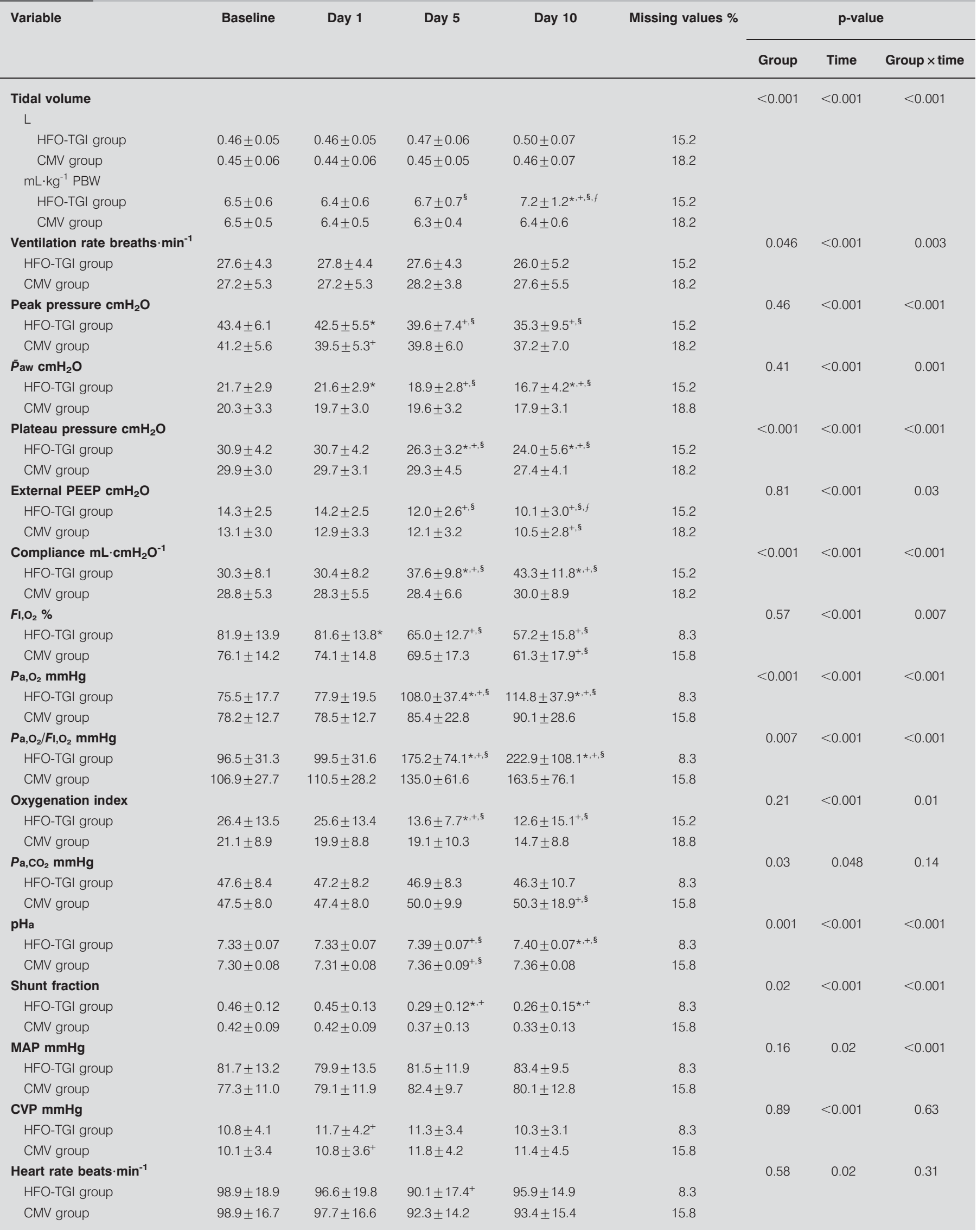




\section{TABLE 4 Continued}

\begin{tabular}{|c|c|c|c|c|c|c|c|c|}
\hline \multirow[t]{2}{*}{ Variable } & \multirow[t]{2}{*}{ Baseline } & \multirow[t]{2}{*}{ Day 1} & \multirow[t]{2}{*}{ Day 5} & \multirow[t]{2}{*}{ Day 10} & \multirow[t]{2}{*}{ Missing values $\%$} & \multicolumn{3}{|c|}{ p-value } \\
\hline & & & & & & Group & Time & Group $\times$ time \\
\hline HFO-TGl group & $4.0 \pm 1.03$ & $4.0 \pm 1.0$ & $3.7 \pm 0.9$ & $3.8 \pm 0.5$ & 12.9 & & & \\
\hline CMV group & $4.1 \pm 0.9$ & $4.2 \pm 0.8$ & $4.0 \pm 0.7$ & $4.1 \pm 1.0$ & 21.1 & & & \\
\hline $\mathrm{DO}_{2} \mathrm{ImL} \cdot \mathrm{min}^{-1} \cdot \mathrm{m}^{-2}$ & & & & & & 0.46 & $<0.001$ & 0.18 \\
\hline $\mathrm{Scv}, \mathrm{O}_{2} \%$ & & & & & & 0.14 & 0.09 & 0.30 \\
\hline HFO-TGI group & $70.7 \pm 8.8$ & $71.4 \pm 8.5$ & $72.8 \pm 5.8$ & $71.6 \pm 8.7$ & 8.3 & & & \\
\hline CMV group & $70.7 \pm 6.6$ & $71.0 \pm 6.7$ & $71.0 \pm 6.0$ & $71.9 \pm 4.3$ & 15.8 & & & \\
\hline Lactate $\mathrm{mmol} \cdot \mathrm{L}^{-1}$ & & & & & & 0.12 & $<0.001$ & 0.32 \\
\hline HFO-TGI group & $2.5 \pm 1.8$ & $2.3 \pm 1.8$ & $1.9 \pm 1.1$ & $1.5 \pm 0.8$ & 8.3 & & & \\
\hline Noradrenaline ${ }^{\top} \mu \mathrm{g} \cdot \mathrm{kg}^{-1} \cdot \mathrm{min}^{-1}$ & & & & & & 0.38 & 0.03 & 0.12 \\
\hline HFO-TGl group & $0.19 \pm 0.16$ & $0.22 \pm 0.23$ & $0.15 \pm 0.13$ & $0.09 \pm 0.11$ & 8.3 & & & \\
\hline CMV group & $0.19 \pm 0.16$ & $0.19 \pm 0.17$ & $0.15 \pm 0.15$ & $0.12 \pm 0.16$ & 15.8 & & & \\
\hline SOFA score & & & & & & 0.02 & $<0.001$ & 0.03 \\
\hline HFO-TGI group & $11.7 \pm 2.7$ & $11.5 \pm 2.7$ & $10.4 \pm 3.7$ & $7.4 \pm 4.6^{*,+, \xi_{,} f}$ & 8.3 & & & \\
\hline CMV group & $12.1 \pm 2.6$ & $11.9 \pm 2.7$ & $11.9 \pm 4.2$ & $10.0 \pm 3.8$ & 15.8 & & & \\
\hline
\end{tabular}

Data are presented as mean $\pm \mathrm{SD}$, unless otherwise stated. Data originate from physiological measurements performed during conventional mechanical ventilation in each one of the 125 patients (intention-to-treat analysis), within $2 \mathrm{~h}$ before randomization (baseline), and between 09:00 and 10:00 h on days 1-10 post-randomisation. Detailed data on physiological endpoints and the Sequential Organ Failure Assessment (SOFA) score are presented in fig. 3. Pressure, volume and respiratory rate values were those displayed by the ventilator. Respiratory compliance was calculated as tidal volume divided by the end-inspiratory to end-expiratory plateau airway pressure difference $[7,12]$. For both groups, analysed data corresponded to 11 consecutive time-points, i.e. at baseline and on the morning of each of the first 10 days post-randomisation. For between-group comparisons at each time-point, we used the Bonferroni correction, i.e. we multiplied the obtained p-values by 11 . The 11 timepoints resulted in a total of 55 within-group pairwise comparisons; thus, we multiplied p-values from within-group comparisons by 55 . The handling of missing values is summarised in the online supplementary material. HFO: high-frequency oscillation; TGl: tracheal gas insufflation; CMV: conventional mechanical ventilation; PBW: predicted body weight; $\bar{P}_{\text {aw: }}$ mean airway pressure; $\mathrm{PEEP}$ : positive end-expiratory pressure; $\mathrm{Fl}, \mathrm{O}_{2}$ : inspiratory oxygen fraction; $\mathrm{Pa}_{3} \mathrm{O}_{2}$ : arterial oxygen tension; $\mathrm{Pa}_{2} \mathrm{CO}_{2}$ : arterial carbon dioxide tension; $\mathrm{pHa}$ : arterial $\mathrm{pH}$; MAP: mean arterial pressure; CVP: central venous pressure; $\mathrm{DO}_{2}$ l: oxygen delivery index; Scv, $\mathrm{O}_{2}$ : central venous oxygen

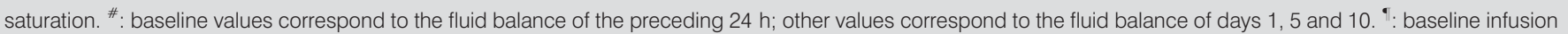
rate was the infusion rate recorded just prior to randomisation; other infusion rates are the average infusion rates of days 1,5 and 10 ; during days $1-10$, all patients of both groups required a noradrenaline infusion for $\geqslant 1 \mathrm{~h}$ to maintain a MAP of $\geqslant 70 \mathrm{mmHg} .{ }^{*}: p<0.05$ versus CMV group at that particular time-point; ${ }^{+}: p<0.05$ versus baseline; ${ }^{\text {s. }} \mathrm{p}<0.05$ versus day $1 ; f: p<0.05$ versus day 5 .

fell to $221.0 \pm 82.3 \mathrm{mmHg}$ (end of stabilisation period) and to $172.2 \pm 33.4 \mathrm{mmHg}$ (weaning period, $30 \mathrm{~min}$ after TGI discontinuation; eFigure 5 in the online supplementary material). The initial $\bar{P}_{\text {aw }}$ was reduced by $6 \mathrm{cmH}_{2} \mathrm{O}$ within $5.5 \pm 0.6$ and $16.3 \pm$ $14.4 \mathrm{~h}$ in 124 and 93 out of 223 HFO-TGI sessions, respectively. HFO-TGI resulted in significant improvements in post- versus presession oxygenation and lung mechanics, and did not affect haemodynamics or arterial carbon dioxide tension versus the preceding CMV (details provided in the text and eFigure 6 of the online supplementary material). Intervention failure (fig. 1) occurred in six sessions (online supplementary material).

\section{Intervention-associated complications}

On days 1-4, HFO-TGI group and CMV group patients received $4.7 \pm 3.5$ and $4.7 \pm 1.5 \mathrm{RMs}$ per day, respectively $(\mathrm{p}=0.79)$; $\mathrm{RM}$ abort rates due to hypotension or desaturation were $\sim 6 \%$ in both groups (online supplementary material). On days 5-10, 19
HFO-TGI group patients received $2.0 \pm 2.2$ RMs per day and the $\mathrm{RM}$ abort rate was $16.5 \%$; this exclusive $\mathrm{RM}$ use had no significant effect on study outcomes (online supplementary material). On HFO-TGI initiation, 10 (16.7\%) patients experienced one RM-associated, major drop in systolic pressure to $75.1 \pm 5.4 \mathrm{mmHg}$ (average drop $28.0 \pm 7.2 \%$ ) and cardiac index to $2.4 \pm 0.6 \mathrm{~L} \cdot \mathrm{min}^{-1} \cdot \mathrm{m}^{-2}$ (average drop $26.0 \pm 11.4 \%$ ). In nine patients, haemodynamic status was restored within $\leqslant 10 \mathrm{~min}$ with fluids and vasopressors. In one patient, a chest tube was inserted for tension pneumothorax. Five patients (three of whom were in the HFO-TGI group) experienced one RMassociated, prolonged (duration 3-5 min) desaturation (maximum absolute drop in oxygen saturation $7-17 \%$ ), which was reversed within $\leqslant 5 \mathrm{~min}$ after $\mathrm{RM}$ discontinuation. In one patient, day 10 bronchoscopy revealed a haemorrhagic posterior tracheal mucosa, suggesting TGI-induced mucosal damage (online supplementary material). 

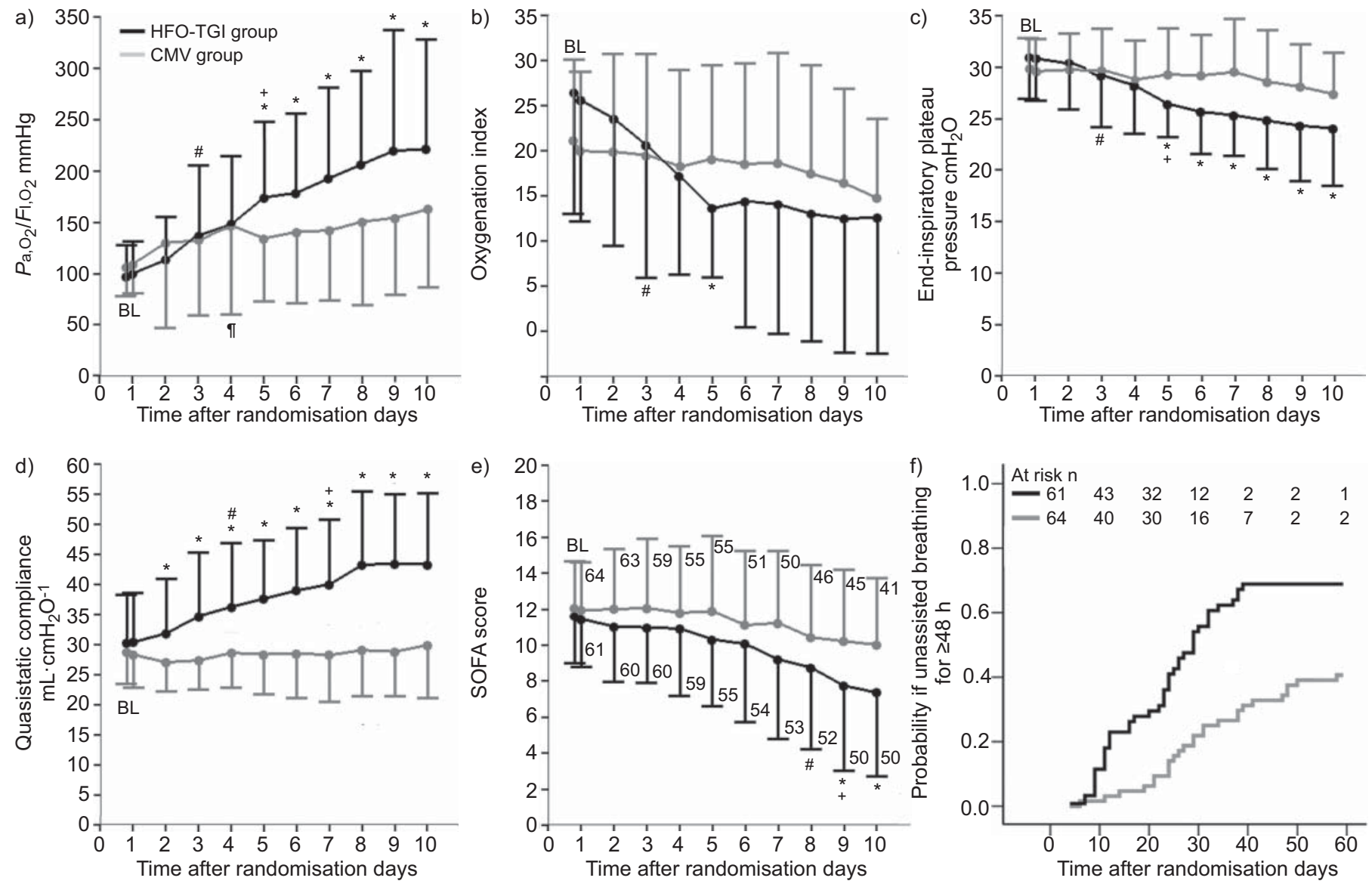

FIGURE 3. Data for a-d) major physiological variables, e) Sequential Organ Failure Assessment (SOFA) score and f) probability of achieving unassisted breathing for $\geqslant 48 \mathrm{~h}$. a-d) Circles represent means of measurements obtained during conventional mechanical ventilation (CMV), within $2 \mathrm{~h}$ before randomisation (baseline (BL)) and between $09: 00$ and 10:00 $\mathrm{h}$ on days 1-10 post-randomisation. e) Circles represent the mean SOFA score at baseline and at the time-points of the aforementioned physiological measurements, and numbers represent surviving patients. One CMV group patient achieved unassisted breathing from day 6 onward. One, two, two and one high-frequency oscillation (HFO) tracheal gas insufflation (TGI) group patients achieved unassisted breathing from days 4, 7, 9 and 10 onward, respectively, and one and one HFO-TGl group patients achieved unassisted breathing for $72 \mathrm{~h}$ starting from day 9 and unassisted breathing solely during day 9 , respectively (table 3). The significant between-group difference observed on days 9 and 10 was partly due to the more frequent development of post-randomisation multiple organ failure in the CMV group (see main text for details). a-e) Error bars represent SD. Summary results for a) arterial oxygen tension $\left(\mathrm{Pa}_{2} \mathrm{O}_{2}\right)$ /inspiratory oxygen fraction $\left(\mathrm{Fl}, \mathrm{O}_{2}\right)$, b) oxygenation index, C) plateau pressure, d) compliance and e) SOFA score are presented in table 4. Between-group $\left(^{*}\right)$ and within-group $\left(^{\left(*^{*},+\right.}\right)$ comparisons were subjected to the Bonferroni correction (see footnote of table 4 and the online supplementary material). *: $p<0.05$ versus CMV group at that particular time-point; ${ }^{*}: p<0.05$ versus baseline maintained from this point onward; $": p<0.05$ versus baseline; ${ }^{+}: p<0.05$ versus day 2 maintained from this point onward. f) $p<0.001$ by log-rank test, which compares the probability of achieving unassisted breathing for $\geqslant 48 \mathrm{~h}$ within days $1-60$ post-randomisation. Assignment to CMV group was an independent predictor of unassisted breathing for $\geqslant 48 \mathrm{~h}$ within days $1-60$ (hazard ratio $0.37,95 \% \mathrm{Cl} 0.22-0.61 ; \mathrm{p}<0.001$ ).

\section{Clinical course data}

On days 1-60, the HFO-TGI group had more ventilator-free days versus the CMV group (median (interquartile range) 31.0 $(0.0-42.0)$ versus $0.0(0.0-23.0)$ days; $\mathrm{p}<0.001)$, and more days without respiratory (46.0 (2.0-54.0) versus 5.0 (0.0-33.8) days; $\mathrm{p}=0.001)$, coagulation $(60.0(21.5-60.0)$ versus 17.0 (5.3-60.0) days; $\mathrm{p}=0.003)$, liver (60.0 (28.5-60.0) versus 24.5 (6.3-60.0) days; $\mathrm{p}=0.003)$, circulatory (43.0 (2.0-55.0) versus 6.5 (0.0-39.0) days; $\mathrm{p}=0.001)$, renal (60.0 (12.0-60.0) versus 15.5 (2.0-60.0) days; $\mathrm{p}=0.001)$ and nonpulmonary organ failure $(29.0(0.0-46.5)$ versus $0.0(0.0-30.8)$ days; $\mathrm{p}=0.001)$; results were similar for days $1-28$ (online supplementary material).

On days 1-10, SOFA score improved in the HFO-TGI group (table 4 and fig. 3e). On days 1-60, the HFO-TGI group had more follow-up days versus the CMV group (60.0 (28.5-60.0) versus 24.5 (7.0-60.0) days; $\mathrm{p}=0.001)$, lower proportions of follow-up days with MOF (11.7\% (1.7-69.1\%) versus $51.0 \%$ (11.3-100.0\%); $\mathrm{p}=0.002)$, less frequent MOF occurrence in patients without MOF at baseline (seven (33.3\%) out of 21 versus 15 (78.9\%) out of 19 subjects; $\mathrm{p}=0.005$ ) (respective times of occurrence mean $\pm \mathrm{SD}$ $4.7 \pm 5.1$ versus $8.5 \pm 6.6$ days post-randomisation; $p=0.20$ ), similar absolute number of days on ventilator $(20.1 \pm 13.3$ versus $20.4 \pm 15.9$ days; $\mathrm{p}=0.90)$, and more patients $(42(68.9 \%)$ out of 61 versus $26(40.6 \%)$ out of 64 patients; $p=0.002)$ achieving unassisted breathing for $\geqslant 48 \mathrm{~h}$ (i.e. successful weaning) in a shorter time $(21.4 \pm 10.0$ versus $30.9 \pm 12.8$ days; $p=0.001)$ (fig. $3 f)$.

Throughout the study period, the HFO-TGI group, versus the CMV group, had $24.3 \pm 20.9$ versus $22.3 \pm 20.0$ total days on a 
ventilator $(\mathrm{p}=0.60)$ and $35.0(18.0-61.5)$ versus $21.0(7.0-57.3)$ total days of in-hospital follow-up $(\mathrm{p}=0.07)$. The HFO-TGI group had comparable percentages of patients with an occurrence of barotrauma as a new pneumothorax versus the CMV group (six $(9.8 \%)$ out of 61 versus nine $(14.1 \%)$ out of 61 patients; $\mathrm{p}=0.59)$, and one or more episodes of ventilator-associated pneumonia (VAP) $(49.2 \%$ versus $50.0 \%$; $>>0.99)$, catheter-related bacteraemia $(21.3 \%$ versus $18.8 \%$; $=0.82)$, Gram-negative sepsis ( $59.0 \%$ versus $48.4 \% ; p=0.28)$, renal $(32.8 \%$ versus $37.5 \%$; $p=0.71)$, coagulation $(24.6 \%$ versus $26.6 \%$; $=0.84)$, hepatic $(9.8 \%$ versus $9.4 \% ; \mathrm{p}>0.99)$ and neurological failure (52.5\% versus $46.9 \%$; $\mathrm{p}=0.59)$, heparin-induced thrombocytopenia $(16.4 \%$ versus $18.8 \% ; \mathrm{p}=0.82)$, failure to maintain unassisted breathing $(47.5 \%$ versus $32.8 \% ; p=0.10)$, and paresis $(18.0 \%$ versus $15.6 \%$; $\mathrm{p}=0.81)$. VAP occurrence was not a predictor of successful weaning but prolonged the mean time to its achievement by $\sim 8-9$ days in both groups (online supplementary material). Further details on complications, and data on administered medication and rescue oxygenation (used in six (9.4\%) out of 64 CMV group patients) are provided in the online supplementary material.

On days 1-28, CMV protocol violations corresponded to $6.3 \%$ versus $3.8 \%$ of the follow-up time in the HFO-TGI group and CMV group, respectively $(p=0.004)$. The HFO-TGI algorithm was applied without deviation in 202 (90.1\%) sessions. The CMV group RM protocol was accurately applied in $98.8 \%$ of the corresponding patient-days. There was no between-group crossover. Study centre did not affect study outcomes (data not shown).

\section{Survival}

Survival to hospital discharge was higher in the HFO-TGI group versus the CMV group (38 (62.3\%) out of 61 versus 23 $(35.9 \%)$ out of 64 patients; $p=0.004$ by Fisher's exact test) (figure 4). There was no significant between-group difference in the ICU and hospital stays of survivors and nonsurvivors (table 5), or the survival of patients with pulmonary contusionassociated ARDS (HFO-TGI group versus CMV group: 13 $(59.1 \%)$ out of 22 versus eight $(66.7 \%)$ out of 12 patients; $\mathrm{p}=0.72$ ) (online supplementary material). Death attributable to MOF [19] was less frequent in the HFO-TGI group versus the CMV group (eight (13.1\%) out of 61 versus 22 (34.4\%) out of 64 patients; $\mathrm{p}=0.006)$ (online supplementary material). Independent predictors of in-hospital mortality included assignment to the CMV group (hazard ratio (HR) 2.64, 95\% CI 1.51-4.61; $\mathrm{p}=0.001)$, baseline arterial blood lactate (HR 1.16, 95\% CI 1.061.28; $\mathrm{p}=0.002)$ and baseline Simplified Acute Physiology Score (SAPS) II (HR 1.04, 95\% CI 1.00-1.06; p=0.003).

\section{DISCUSSION}

We showed an increased efficacy of intermittent HFO-TGI recruitment sessions in early (exhibiting high likelihood of lung recruitability) and severe ARDS. During the recruitment period, the 2.3-fold average $\mathrm{Pa}, \mathrm{O}_{2} / \mathrm{FI}_{1} \mathrm{O}_{2}$ rise was consistent with enhanced lung recruitment $[6-10,20]$. This enabled reduction of the initial respiratory system distending pressure by $6 \mathrm{cmH}_{2} \mathrm{O}$ (stabilisation period), with maintenance of $\sim 85 \%$ of the oxygenation benefit. The evolution of compliance (fig. 3d) suggests progressive increase in aerated lung volume [20], which explains the concurrent plateau-pressure reduction (fig. 3c). These changes imply prompt inhibition of the

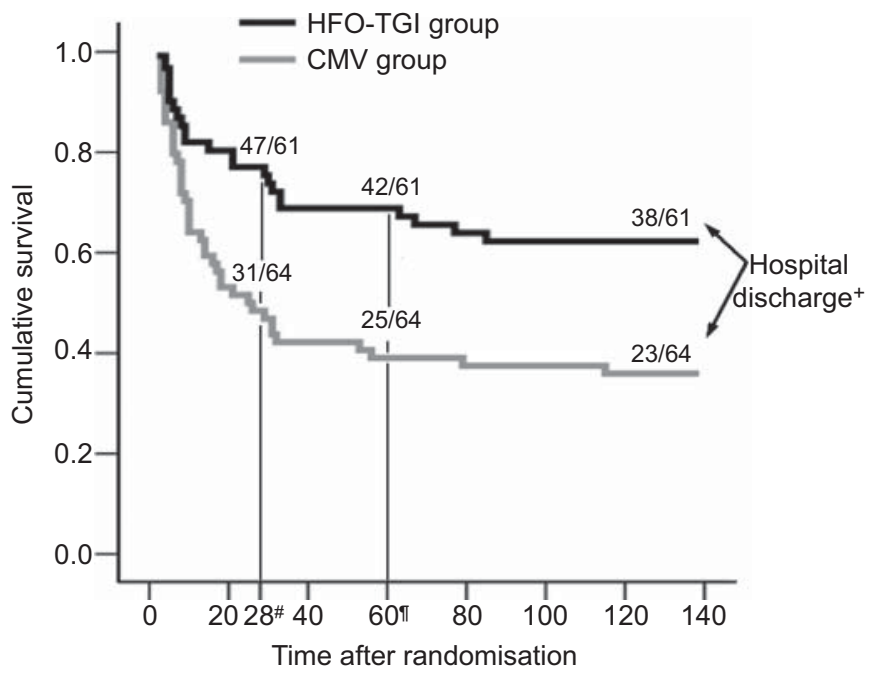

FIGURE 4. Survival to hospital discharge. $p=0.002$ by log-rank test; $p=0.004$ by Fisher's exact test. HFO: high-frequency oscillation; TGI: tracheal gas insufflation; CMV; conventional mechanical ventilation. \#: $p=0.002$ by Fisher's exact test for 28 -day survival. ${ }^{\bullet}: p=0.001$ by Fisher's exact test for 60 -day survival ${ }^{+}$: for patients with hospital stays of $\geqslant 60$ days, follow-up was terminated at the timepoint of hospital discharge; the actual range of hospital stay was 17-137 days; patients discharged before day 60 (HFO-TGl group, $n=25$; CMV group, $n=11$ ) were followed as outpatients until day 60; the surviving status of all 61 discharged patients at 150 days post-randomisation was reconfirmed through telephone communication.

injurious mechanical stresses to the lung [13, 21], leading to prevention of biotrauma-associated organ injury [21] and improved survival.

In the CMV group, the absence of physiological improvements (fig. 3a-d) was associated with prolonged and multiple organ dysfunction during follow-up and a long-term mortality of $64.1 \%$ [19].

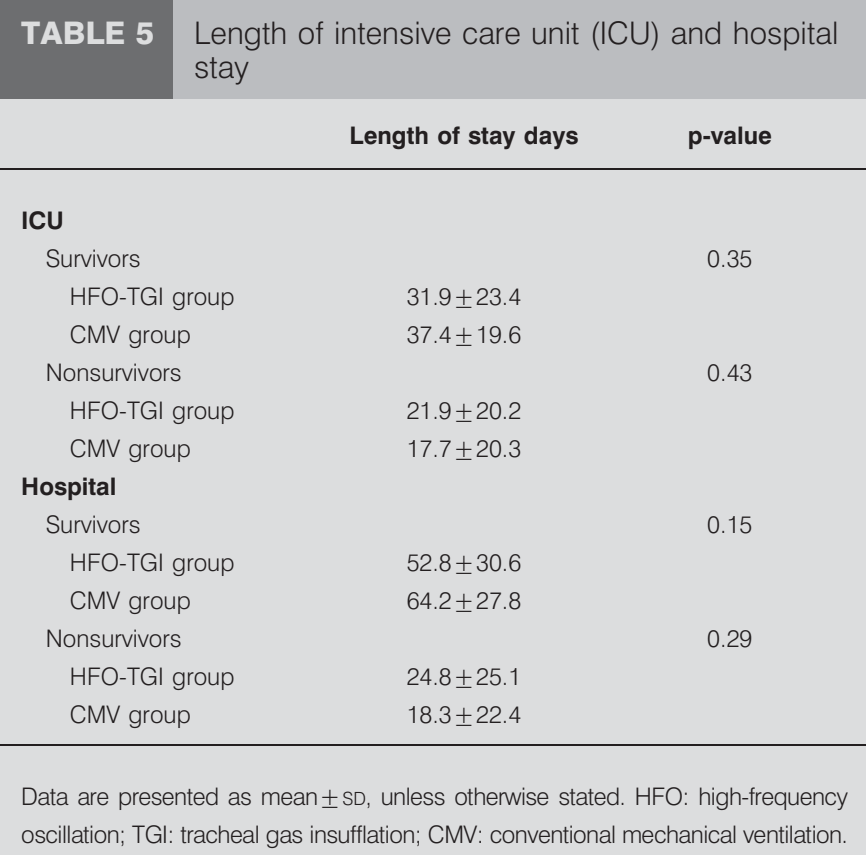


In a recent multicentre study [22], ARDS patients with similar baseline SAPS II scores and oxygenation disturbances had similar evolution of their respiratory variables and SOFA scores during early follow-up, and a long-term mortality of $63.2 \%$.

Previous trials evaluated continuous HFO [23, 24], prone positioning [22, 25, 26] and high PEEP with/without RMs [27-29]. Positive findings comprised improved oxygenation [22, 24-29], improved respiratory mechanics [26, 27, 29], lower rates of refractory hypoxaemia [28, 29], and more ventilatorfree and organ failure-free days [29]. However, results on mortality were inconclusive. In contrast, our results on both physiology and outcome favour intermittent recruitment with HFO-TGI and RMs. This suggests improved lung protection throughout the early phase of ARDS through a more effective method of periodic lung recruitment.

We compared a recruitment strategy of combined HFO, TGI and short-lasting RMs to short-lasting RMs alone during lungprotective CMV. Theoretically, longer-lasting RMs could have produced different results. However, the best way to perform RMs still remains undetermined. Also, TGI usefulness is still unproven, and similar outcome results might have been obtained with an HFO-RM recruitment protocol. Nevertheless, three physiological studies suggest a TGI-related, gas-exchange and/or lower lung recruitment benefit [7, 8, 30]. Furthermore, the present study's potentially nonprotective HFO settings may augment lung base recruitment $[8,30]$.

During days 1-10, the study protocol was applied by subgroups of two investigators assigned to each patient of each group on a rotating $12-\mathrm{h}$ basis. There was tighter tidal volume control (table 4) and accurate RM protocol application in the CMV group. Medical treatment (including sedation/paralysis) was similar in both groups (online supplementary material). Notable, but promptly/effectively treated, complications occurred in 13 (5.8\%) out of the 223 HFO-TGI sessions (see Results section).

\section{Limitations}

Our sample size was relatively small, but the study was adequately powered to detect a substantial survival benefit. The study design was unblinded and the results originate from just two centres, thus warranting further multicentre confirmation. Also, the study was conducted over two periods, primarily for feasibility reasons (online supplementary material). Lastly, although the high CMV group mortality and small number of ventilator-free days may be justifiable by disease severity, a selection bias in favour of the HFO-TGI group cannot be totally excluded.

Another limitation was the lack of measurement of proinflammatory cytokines during the intervention period. However, the causal link among persistence of ARDS, systemic inflammation and development of multiple organ dysfunction/ MOF is well-established [31]. Furthermore, our physiological and SOFA score results (fig. 3) are consistent with this sequence of events occurring more frequently in the CMV group, with a consequent increase in the probability of death [19].

Pre-enrolment duration of mechanical ventilation (DMV) was variable (table 2), with a potentially unpredictable impact on patient outcomes [30, 32]. Indeed, although pre-enrolment DMV exceeded 7 days [33] in just 12 (9.6\%) patients (eight in the HFO-TGI group), the results of a recent multicentre trial imply that any difference in the overall management strategy of early ARDS might affect results for mortality [34].

\section{Conclusions}

Our two-centre results suggest that in early/severe ARDS, the addition of recruitment sessions of HFO-TGI with RMs to lungprotective CMV may improve survival to hospital discharge. This is supported by the associated improvements in respiratory physiology, ventilator-free days and nonpulmonary organ function.

\section{SUPPORT STATEMENT}

This study was funded by the Thorax Foundation (Athens, Greece; www.thorax-foundation.gr) and the Project "Synergasia" (Cooperation)

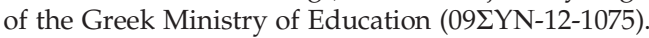

\section{CLINICAL TRIAL}

This study is registered at www.clinicaltrials.gov with identifier numbers NCT00416260 (first period) and NCT00637507 (second period).

\section{STATEMENT OF INTEREST}

None declared.

\section{ACKNOWLEDGEMENTS}

First study period results (www.clinicaltrials.gov identifier NCT00416260) were presented in part at 1) the 11th State-of-the-Art Interdisciplinary Review Course (Athens, Greece; April 20-22, 2007), and 2) the 20th (Berlin, Germany; 2007) and 22nd (Vienna, Austria; 2009) annual congresses of the European Society of Intensive Care Medicine [35, 36]. Also, a lecture based on the aforementioned results was given at the 2008 International Symposium on Intensive Care and Emergency Medicine in Brussels, Belgium. The results of the first period have also been summarised in a recently published meta-analysis [37]. The Study Protocol can be accessed at the official website of the Scientific Society of Evaggelismos Hospital [38].

The Study Chairpersons were S.D. Mentzelopoulos (principal investigator), S. Malachias (principal investigator), S.G. Zakynthinos (study director), C. Roussos (study chair) and E. Zakynthinos (collaborating centre principal investigator). The members of the Independent Main Endpoint and Safety Monitoring Committee were P. Politis, E. Stamataki and Z. Mastora (all Evaggelismos Hospital), and Z. Daniil (Larissa University Hospital). Overall study and data quality assurance was performed by P. Politis, E. Stamataki, Z. Mastora and Z. Daniil.

We wish to thank to P. Zygoulis for his assistance with the study protocol and patient follow-up at Larissa University hospital. We also wish to thank M. Tzoufi (Dept of Intensive Care Medicine, Evaggelismos Hospital, Athens, Greece) for her assistance in the analyses and presentation of the study results. The representative of Sensormedics in Greece is Meditrust A.E.

\section{REFERENCES}

1 Fessler HE, Derdak S, Ferguson ND, et al. A protocol for highfrequency oscillatory ventilation in adults: results from a roundtable discussion. Crit Care Med 2007; 35: 1649-1654.

2 Chan KP, Stewart TE, Mehta S. High-frequency oscillatory ventilation for adult patients with ARDS. Chest 2007; 131: 1907-1916.

3 Hager DN, Fessler HE, Kaczka DW, et al. Tidal volume delivery during high-frequency oscillatory ventilation in adults with acute respiratory distress syndrome. Crit Care Med 2007; 35: 1522-1529.

4 Imai Y, Slutsky AS. High-frequency oscillatory ventilation and ventilator-induced lung injury. Crit Care Med 2005; 33: S129-S134.

5 Ferguson ND, Slutsky AS. Point: High-frequency ventilation is the optimal physiological approach to ventilate ARDS patients. J Appl Physiol 2008; 104: 1230-1231.

6 Ferguson ND, Chiche JD, Kacmarek RM, et al. Combining highfrequency oscillatory ventilation and recruitment in adults with 
early acute respiratory distress syndrome: The Treatment with Oscillation and an Open Lung Strategy (TOOLS) Trial pilot study. Crit Care Med 2005; 33: 479-486.

7 Mentzelopoulos SD, Roussos C, Koutsoukou A, et al. Acute effects of combined high-frequency oscillation and tracheal gas insufflation in severe acute respiratory distress syndrome. Crit Care Med 2007; 35: 1500-1508.

8 Mentzelopoulos SD, Malachias S, Kokkoris S, et al. Comparison of high frequency oscillation and tracheal gas insufflation versus standard high frequency oscillation at two levels of tracheal pressure. Intensive Care Med 2010; 36: 810-816.

9 Dolan S, Derdak S, Solomon D, et al. Tracheal gas insufflation combined with high-frequency oscillatory ventilation. Crit Care Med 1996; 24: 458-465.

10 Nahum A. Tracheal gas insufflation. Crit Care 1998; 2: 43-47.

11 Hager DN, Krishnan JA, Hayden DL, et al. Tidal volume reduction in patients with acute lung injury when plateau pressures are not high. Am J Respir Crit Care Med 2005; 172: 1241-1245.

12 Mentzelopoulos SD, Roussos C, Zakynthinos SG. Prone position reduces lung stress and strain in severe acute respiratory distress syndrome. Eur Respir J 2005; 25: 534-544.

13 Gattinoni L, Carlesso E, Cadringher P, et al. Physical and biological triggers of ventilator-induced lung injury and its prevention. Eur Respir J 2003; 22: Suppl. 47, 15s-25s.

14 Ventilation with lower tidal volumes as compared with traditional tidal volumes for acute lung injury and the acute respiratory distress syndrome. The Acute Respiratory Distress Syndrome Network. N Engl J Med 2000; 342: 1301-1308.

15 Bernard GR, Artigas A, Brigham KL, et al. The AmericanEuropean Consensus Conference on ARDS. Definitions, mechanisms, relevant outcomes, and clinical trial coordination. Am J Respir Crit Care Med 1994; 149: 818-824.

16 Esteban A, Anzueto A, Frutos F, et al. Characteristics and outcomes in adult patients receiving mechanical ventilation: a 28-day international study. JAMA 2002; 287: 345-355.

17 Grasso S, Mascia L, Del Turco M, et al. Effects of recruiting maneuvers in patients with acute respiratory distress syndrome ventilated with protective ventilatory strategy. Anesthesiology 2002; 96: 795-802.

18 Vincent JL, de Mendonça A, Cantraine F, et al. Use of the SOFA score to assess the incidence of organ dysfunction/failure in intensive care units: Results of a multicenter prospective study. Crit Care Med 1998; 26: 1793-1800.

19 Ferring M, Vincent JL. Is outcome from ARDS related to the severity of respiratory failure? Eur Respir J 1997; 10: 1297-1300.

20 Henzler D, Pelosi P, Dembinski R, et al. Respiratory compliance but not gas exchange correlates with changes in lung aeration after a recruitment maneuver: an experimental study in pigs with saline lavage acute lung injury. Crit Care 2005; 9: R471-R482.

21 Plötz FB, Slutsky AS, van Vught AJ, et al. Ventilator-induced lung injury and multiple system organ failure: a critical review of facts and hypotheses. Intensive Care Med 2004; 30: 1865-1872.

22 Taccone P, Pesenti A, Latini R, et al. Prone positioning in patients with moderate and severe acute respiratory distress syndrome. A randomized controlled trial. JAMA 2009; 302: 1977-1984.
23 Bollen CW, van Well GT, Sherry T, et al. High frequency oscillatory ventilation compared with conventional mechanical ventilation in adult respiratory distress syndrome: a randomized controlled trial [ISRCTN24242669]. Crit Care 2005; 9: R430-R439.

24 Derdak S, Mehta S, Stewart TE, et al. High-frequency oscillatory ventilation for acute respiratory distress syndrome in adults: a randomized, controlled trial. Am J Respir Crit Care Med 2002; 166: 801-808.

25 Gattinoni L, Tognoni G, Pesenti A, et al. Effect of prone positioning on the survival of patients with acute respiratory failure. $N$ Engl J Med 2001; 345: 568-573.

26 Mancebo J, Fernández R, Blanch L, et al. A multicenter trial of prolonged prone ventilation in severe acute respiratory distress syndrome. Am J Respir Crit Care Med 2006; 173: 1233-1239.

27 Brower RG, Lanken PN, MacIntyre N, et al. Higher versus lower positive end-expiratory pressures in patients with the acute respiratory distress syndrome. N Engl J Med 2004; 351: 327-336.

28 Meade MO, Cook DJ, Guyatt GH, et al. Ventilation strategy using low tidal volumes, recruitment maneuvers, and high positive endexpiratory pressure for acute lung injury and acute respiratory distress syndrome: a randomized controlled trial. JAMA 2008; 299: 637-645.

29 Mercat A, Richard JC, Vielle B, et al. Positive end-expiratory pressure setting in adults with acute lung injury and acute respiratory distress syndrome: a randomized controlled trial. JAMA 2008; 299: 646-655.

30 Mentzelopoulos SD, Theodoridi M, Malachias S, et al. Scanographic comparison of high frequency oscillation with versus without tracheal gas insufflation in acute respiratory distress syndrome. Intensive Care Med 2011; 37: 990-999.

31 Meduri GU, Annane D, Chrousos GP, et al. Activation and regulation of systemic inflammation in ARDS: rationale for prolonged glucocorticoid therapy. Chest 2009; 136: 1631-1643.

32 Monchi M, Bellenfant F, Cariou A, et al. Early predictive factors of survival in the acute respiratory distress syndrome. A multivariate analysis. Am J Respir Crit Care Med 1998; 158: 1076-1081.

33 Klompas M, Khan Y, Kleinman K, et al. Multicenter evaluation of a novel surveillance paradigm for complications of mechanical ventilation. PLoS One 2011; 6: e18062.

34 Papazian L, Forel JM, Gacouin A, et al. Neuromuscular blockers in early acute respiratory distress syndrome. N Engl J Med 2010; 363: 1107-1116.

35 Abstracts of the 20th Annual Congress of the European Society of Intensive Care Medicine, 7-10 October 2007, Berlin, Germany. Intensive Care Med 2007; 33: Suppl. 2, S5-S271.

36 Abstracts of the 22nd Annual Congress of the European Society of Intensive Care Medicine (ESICM). Vienna, Austria. October 11-14, 2009. Intensive Care Med 2009; 35: Suppl. 1, S5-S306.

37 Sud S, Sud M, Friedrich JO, et al. High frequency oscillation in patients with acute lung injury and acute respiratory distress syndrome (ARDS): systematic review and meta-analysis. BMJ 2010; 340: c2327.

38 Scientific Society of Evangelismos Hospital. The Scientific Acitivities of SSEH. www.sseh.gr/Education/educationtext.php Date last accessed: January 3, 2012. Date last updated: March 7, 2008. 